\title{
SOME POINT-SET PROPERTIES AND THE EDGE PATH GROUP OF A GENERALIZED UNIFORM SPACE
}

\author{
BY \\ ROBERT W. DEMING
}

1. Introduction and summary of results. Lubkin [6] has defined the category $\mathscr{S}$ of spaces and contiguous functions. A space is a set $X$ with a contiguity (filter of entourages in $X$ ), and if $X$ and $Y$ are spaces, a function $f: X \rightarrow Y$ is contiguous provided that for each entourage $v \in$ contig $Y, f\left(u_{x}\right) \subset v_{f(x)}$ for some $u \in$ contig $X$ for all $x \in X$. $\left(u_{x}=\{y \in X:(x, y) \in u\}\right.$.) Thus the categories $\mathscr{U}$ of uniform spaces and $\mathscr{Q}$ of quasi-uniform spaces (Pervin [7]) are subcategories of $\mathscr{S}$. Moreover, Carver [2] had defined the category $\mathscr{P} \subset \mathscr{S}$ of pseudo-uniform spaces. Since a quasi-uniform space is a pseudo-uniform space (Carver [2] and Theorem 2.1) the hierarchy $\mathscr{U} \subset \mathscr{Q} \subset \mathscr{P} \subset \mathscr{S}$ of subcategories of $\mathscr{S}$ results.

The method of generating topologies from uniformities is applicable to generating a topology $T(C)$ from a contiguity $C$. The assignments $(X, C) \rightarrow(X, T(C))$ and $f \rightarrow f$ is a functor $T: \mathscr{S} \rightarrow \mathscr{T}$ where $\mathscr{T}$ is the category of topological spaces. Several examples of functors in a class $\mathscr{F}$ of functors $F: \mathscr{T} \rightarrow \mathscr{S}$ such that $T F=1$ are given in $\S 2$. Some of the properties of these functors are examined in $\$ 2$ also. For instance, each functor in $\mathscr{F}$ preserves the property of connectedness (although $T$ does not).

If $u$ is an entourage in $X, x, y \in X$ then a $u$-edge path from $x$ to $y$ is a finite sequence $x(1), x(2), \ldots, x(n)$ of points in $X$ such that $x(1)=x, x(n)=y$ and $u_{x(i)} \cap u_{x(i+1)} \neq \varnothing$ for $i=1, \ldots, n-1$. Thus a space $(X, C)$ is connected iff for each pair $x, y \in X$ and each $u \in C$ there is a $u$-edge path from $x$ to $y$ (Lubkin [6]). In §3, an equivalence relation is defined on the set of $u$-edge paths from $x$ to $y$. An edge path from $x$ to $y$ is a collection $\left\{a_{u}: u \in C\right\}$ where $a_{u}$ is a $u$-edge path from $x$ to $y$ and such that for $u, v \in C, u \subset v, a_{u}$ and $a_{v}$ are equivalent $v$-edge paths. ( $a_{u}$ is a priori a $v$-edge path.) Two edge paths from $x$ to $y$ are equivalent iff they are equivalent component-wise. The formation of the edge path group $E\left(X, x_{0}\right)$ of a pointed space $\left(X, C, x_{0}\right)$ is similar to that of $\pi_{1}\left(X, x_{0}\right)$ in a topological setting ( $\left.\S 4\right)$. Moreover, a contiguous function $f:\left(X, x_{0}\right) \rightarrow\left(Y, y_{0}\right)$ induces a homomorphism $f^{*}: E\left(X, x_{0}\right) \rightarrow E\left(Y, y_{0}\right)$ in such a way that the assignments $\left(X, C, x_{0}\right) \rightarrow E\left(X, x_{0}\right), f \rightarrow f$ define a covariant functor $E$ from the category of pointed spaces to the category of groups. Some examples of edge path groups and several counterexamples are given in $\$ 5$.

The functor $E$ can be composed with functors from $\mathscr{F}$ to obtain a family of covariant functors from the category of pointed topological spaces to the category

Presented to the Society, January 26, 1966 under the title, The edge-path group and Cech homology groups of a generalized uniform space; received by the editors July 18, 1966. 
of groups. In $\S 6$ the compositions of $E$ with the functors given as examples in $\S 2$ are examined. The existence of a natural transformation from the functor $\pi_{1}$ to each of these compositions is shown, and homotopic invariance is discussed. In addition, the restriction of these compositions to the subcategory of compact metric spaces, for example, are shown to be naturally equivalent functors.

In $\$ 7$, a Čech-type absolute homology sequence is developed for a pseudouniform space $X$ utilizing the open covers $\left\{u_{x}: x \in X\right\}$ associated with open entourages $u$. The main result of $\S 7$ is the existence of an Hurewicz-type homomorphism $E\left(X_{u}, x_{0}\right) \rightarrow H_{1}(u)$ where $X_{u}$ is the space consisting of the set $X$ with the contiguity generated by the single entourage $u \in$ contig $X$, and $H_{1}(u)$ is the first homology group associated with the entourage $u$.

This paper is based on the author's doctoral dissertation presented at New Mexico State University in 1965 . He is indebted to Professor John D. Thomas for many suggestions that have been incorporated herein.

2. Subcategories of $\mathscr{S}$ and functors from $\mathscr{T}$ to $\mathscr{S}$. Let $C$ be a contiguity for $X$. An entourage $u \in C$ is open (closed) relative to $C$ iff $u_{x}$ is open (closed) relative to the topology $T(C)$ for each $x \in X$. Thus, $u$ is open iff for each $x \in X$ and each $y \in u_{x}$ there is a $v \in C$ such that $v_{y} \subset u_{x}$. A contiguity is a pseudo-uniformity provided that it has a base of open entourages.

THEOREM 2.1. A quasi-uniformity is a pseudo-uniformity.

Proof. A quasi-uniformity $C$ for $X$ has a base of entourages of the form $u \circ u$ where $u \in C$. Then for each $w \in C$, there is a sequence $w^{0}, w^{1}, w^{2}, \ldots$ of members of $C$ such that $w^{0}=X \times X, w^{1}=w$ and $w^{n} \circ w^{n} \circ w^{n} \subset w^{n-1}$ for $n=1,2,3, \ldots$ The existence of such a sequence implies the existence of a nonnegative real-valued function $d$ on $X \times X$ such that $d(x, y)+d(y, z) \geqq d(x, z)$ for all $x, y, z \in X$ and such that $w^{0} \supset v(1) \supset w^{1} \supset v(2) \supset \ldots$ where $v(i)=\left\{(x, y): d(x, y)<2^{-i}\right\}$ (Kelley [5, Lemma 6.12 , p. 185]). Thus each of the $v(i)$ 's is in $C$ and if $y \in v(2)_{x}$, select a positive integer $p$ such that $v(p)_{y} \subset v(2)_{x}$. Thus $v(2)$ is an open entourage contained in $w$.

EXAMPLE 2.1 (CARVER [2]). There are contiguities which are not pseudo-uniformities. Let $X$ be the set of real numbers and $C$ be the collection of all entourages $u$ of the form: $u_{x}$ is a neighborhood of $x$ in the usual topology if $x$ is irrational and $u_{x}$ is the set of rationals if $x$ is rational.

Moreover, there are pseudo-uniformities which are not quasi-uniformities (Examples 2.2, 2.4).

If $C$ is a pseudo-uniformity for $X$, then the topology $T(C)$ generated by $C$ is $T_{1}$ iff $\bigcap u=\Delta$, and is Hausdorff iff $\bigcap u^{-1} \circ u=\Delta$ where each intersection is taken over all $u \in C$. If $C$ is a uniformity then $\bigcap u=\Delta$ is equivalent to $\bigcap u^{-1} \circ u=\Delta$ which is consistent with the well-known fact that $T_{1}$ and Hausdorff are equivalent for uniform topologies (Kelley [5, p. 180]).

If $u$ is an entourage in the contiguity $C$ for $X$ then $\mathrm{Cl}(u)($ closure of $u$ ) is that 
entourage in $C$ for which $\mathrm{Cl}(u)_{x}=\mathrm{Cl}\left(u_{x}\right)$ relative to the topology $T(C)$ for each $x \in X$.

Proposition 2.2. If $(X, C)$ is a pseudo-uniform space, then $\mathrm{Cl}(u)=\bigcap w^{-1} \circ u$ : $w \in C$.

Proof. If $x \in X$, then $y$ is a limit point of $u_{x}$ iff $w_{y} \cap u_{x} \neq \varnothing$ for all $w \in C$ iff $(x, y) \in w^{-1} \circ u$ for all $w \in C$ iff $y \in \bigcap\left(w^{-1} u\right)_{x}: w \in C=\left(\bigcap w^{-1} u: w \in C\right)_{x}$.

Proposition 2.3. Let $(X, C)$ be a pseudo-uniform space. If $(X, T(C))$ is regular, then $\bigcap u^{-1} \circ u=\bigcap u$ where each intersection is over $C$.

Proof. $\bigcap u \subset \bigcap u^{-1} \circ u$ and regularity of $T(C)$ implies $\bigcap(\mathrm{Cl} u)_{x} \subset \bigcap u_{x}$ for each $x \in X$. Moreover, $y \in\left(u^{-1} \circ u\right)_{x}$ for all $u \in C$ implies that $y \in\left[(w \cap u)^{-1}\right.$ 。 $(w \cap u)]_{x}$ for all $w \in C$. Thus $y \in\left(w^{-1} \circ u\right)_{x}$ for all $w \in C$, and hence $y \in(\mathrm{Cl}(u))_{x}$ for all $u \in C$. Thus $\left(\bigcap u^{-1} \circ u\right)_{x} \subset(\bigcap u)_{x}$ for each $x \in X$.

Proposition 2.3 is consistent with the fact that regularity and $T_{1}$ together imply Hausdorff.

Let $(X, \tau)$ be a topological space and $\Omega(X, \tau)$ be the collection of all contiguities $C$ for $X$ such that $T(C)=\tau$. Then $\Omega(X, \tau)$ is not empty for every topology $\tau$; in fact, Pervin [7] has shown that $\Omega(X, \tau)$ always contains a quasi-uniformity. The following examples of elements of $\Omega(X, \tau)$ are used several times in the remaining sections of this paper.

EXAMPLE 2.2 (LUBKIN [6]). Let $P(\tau)$ be the contiguity consisting of all entourages $u$ for which $u_{x}$ is a $\tau$-neighborhood of $x . P(\tau)$ is the largest pseudo-uniformity in $\Omega(X, \tau)$ and is not a quasi-uniformity.

EXAMPLe 2.3 (PeRvin [7]). For each open set $S \in \tau$, let $u(S)$ be the entourage in $X$ for which $u(S)_{x}=S$ if $x \in S, u(S)_{x}=X$ if $x \notin S$. The collection $\{u(S): S \in \tau\}$ is a subbase for a quasi-uniformity $Q(\tau) \in \Omega(X, \tau)$.

EXAMPLE 2.4. For each $\tau$-neighborhood $S_{x}$ of $x \in X$, let $u\left(S_{x}\right)$ be the entourage in $X$ for which $u\left(S_{x}\right)_{y}=S_{x}$ if $y=x, u\left(S_{x}\right)_{y}=X$ if $y \neq x$. The collection $\left\{u\left(S_{x}\right): x \in X\right.$, $S_{x}$ is a $\tau$-neighborhood of $\left.x\right\}$ is a subbase for a pseudo-uniformity $P_{0}(\tau) \in \Omega(X, \tau)$. Moreover, $P_{0}(\tau)$ is the smallest pseudo-uniformity in $\Omega(X, \tau)$.

EXAMPLE 2.5. Suppose $(X, \tau)$ is completely regular. The following uniformity is of particular significance below. Let $M(X, I)$ be the set of continuous functions $X \rightarrow I$ where $I$ is the unit interval with the usual topology. For $\alpha \in M(X, I)$ and $\varepsilon>0$ let $u(\alpha ; \varepsilon)=\{(x, y) \in X \times X:|\alpha(x)-\alpha(y)|<\varepsilon\}$. The collection $\{u(\alpha ; \varepsilon)$ : $\alpha \in M(X, I), \varepsilon>0\}$ is a base for a uniformity $U(\tau) \in \Omega(X, \tau)$.

If $(X, \tau)$ and $(Y, \sigma)$ are topological spaces and $f: X \rightarrow Y$ is continuous relative to $\tau$ and $\sigma$, then $f$ is contiguous relative to $P(\tau)$ and $P(\sigma)$ (Example 2.2), $Q(\tau)$ and $Q(\sigma)$ (Example 2.3), and, if $\tau$ and $\sigma$ are completely regular, $U(\tau)$ and $U(\sigma)$ (Example 2.5). Thus, if

$$
\begin{aligned}
& P(X, \tau)=(X, P(\tau)) \text { and } \quad P(f)=f \\
& Q(X, \tau)=(X, Q(\tau)) \text { and } \quad Q(f)=f, \\
& U(X, \tau)=(X, U(\tau)) \text { and } \quad U(f)=f
\end{aligned}
$$


then $P$ and $Q$ are covariant functors $\mathscr{T} \rightarrow \mathscr{S}$ and $U$ is a functor from the subcategory of completely regular topological spaces to $\mathscr{S}$. If $f: X \rightarrow Y$ is a nontrivial, infinite to one continuous function relative to $\tau$ and $\sigma$, then $f$ cannot be contiguous relative to $P_{0}(\tau)$ and $P_{0}(\sigma)$; thus a functor cannot be derived from Example 2.4.

If $\left\{\left(X_{\alpha}, C_{\alpha}\right): \alpha \in I\right\}$ is a collection of spaces, the product contiguity $C$ for $X=\prod X_{\alpha}$ is the smallest contiguity for which every projection $p_{\alpha}: X \rightarrow X_{\alpha}$ is contiguous. $C$ has a base of entourages $u$ such that $u_{x}=\bigcap u(\alpha)_{x(\alpha)}$ where $u(\alpha) \in C_{\alpha}$ is $X_{\alpha} \times X_{\alpha}$ for all but finitely many $\alpha \in I$. Let $\tau$ be the product topology for $X$ derived from the topologies $T\left(C_{\alpha}\right)$. If each of the $C_{\alpha}$ 's is a pseudo-uniformity then $T(C)=\tau$. In this sense, the functor $T$ restricted to the subcategory $\mathscr{P}$ preserves products. Similarly, $T$ restricted to $\mathscr{P}$ preserves category-theoretic subspaces and quotients.

Let $\mathscr{F}$ be the collection of functors $F: \mathscr{T} \rightarrow \mathscr{S}$ such that $T F=1$. (The functors $P$ and $Q$ defined above are in $\mathscr{F}$.) The functor $P \in \mathscr{F}$, for example, preserves quotients and subspaces; however, $P$ does not preserve products.

EXAMPLE 2.6. Let $I$ be the unit interval with the usual topology and let $\tau$ be the product topology for $I \times I$. Let $S(x, y ; \varepsilon)$ be the open sphere with radius $\varepsilon$ about $(x, y) \in I \times I$, and $u$ be the entourage such that $u_{(x, y)}=S(x, y ; 1)$ if $(x, y) \in \Delta$ and $u_{(x, y)}=S(x, y ;|x-y|)$ if $(x, y) \notin \Delta$. Then $u \in P(\tau)$ but $u$ is not in the product contiguity generated from $P(\sigma)$ where $\sigma$ is the usual topology for $I$.

REMARK. If $\left\{\left(X_{\alpha}, \tau_{\alpha}\right)\right\}$ is a collection of compact, regular topological spaces, then the product $(X, \tau)$ is compact and regular. Since in this case there is one and only one uniformity in $\Omega(X, \tau), U(\tau)=C$ where $C$ is the product uniformity generated by the uniformities $U\left(\tau_{\alpha}\right)$.

REMARK. For any collection $\left\{\left(X_{\alpha}, \tau_{\alpha}\right)\right\}$ of topological spaces and any functor $F \in \mathscr{F}$, we have $F(\tau) \supset C$ since each projection $p_{\alpha}: X \rightarrow X_{\alpha}$ is contiguous relative to the contiguities $F(\tau)$ and $F\left(\tau_{\alpha}\right)$.

Let $(X, C)$ be a space, $x \in X$, and $u \in C$. The $u$-component of $x$ is the set points $y \in X$ such that there is a $u$-edge path from $x$ to $y$ (Definition, $\S 1$ ). The quasicomponent of $x$ is the intersection over all $u \in C$ of the $u$-components of $x .(X, C)$ is connected provided that the quasi-component of a point $x \in X$ is $X$. Thus a space is connected iff for every pair $x, y \in X$ and every $u \in C$, there is a $u$-edge path from $x$ to $y .(X, C)$ is locally connected iff $C$ has a base $B$ such that for each $u \in B, u_{x}$ is a connected subspace for all $x \in X$. The uniform space of rational numbers is connected. The functor $T$, therefore, does not preserve the property of connectedness. Indeed, if $(X, \tau)$ is any topological space such that the cardinality of $X$ is infinite, then $\left(X, P_{0}(\tau)\right)$ (Example 2.4) is connected.

A contiguity $C$ for $X$ is discrete provided that for each $x \in X$, there is a $u \in C$ such that $u_{x}=\{x\}$ and $u_{y}=X-\{x\}$ for all $y \in X$ with $y \neq x$. We note that

1. If $C$ is discrete, then $T(C)$ is the discrete topology.

2. If $(X, \tau)$ is a discrete topological space with card $(X)$ infinite, then $P_{0}(\tau)$ is not discrete although $T\left(P_{0}(\tau)\right)=\tau$ is discrete.

3. If $(X, \tau)$ is a discrete topological space, there is a unique smallest discrete 
contiguity in $\Omega(X, \tau)$ (generated by the subbase of entourages of the form $u_{x}=\{x\}, u_{y}=X-\{x\}$ if $\left.y \neq x\right)$.

REMARK. The requirement " $u_{y}=X-\{x\}$ " in the definition of a discrete contiguity insures that a discrete space is not connected.

THEOREM 2.4. Let $(X, \tau)$ be a topological space and $F \in \mathscr{F} . F(\tau)$ is a discrete contiguity iff $\tau$ is the discrete topology.

Proof. If $F(\tau)$ is discrete, then $\tau=T F(\tau)$ is discrete. Suppose $\tau$ is discrete. Let $x, a, b \in X$ with $a \neq b$. Let the function $f_{x}: X \rightarrow X$ be defined such that $f_{x}(y)=\{a\}$ if $y=x$ and $f_{x}(y)=\{b\}$ if $y \neq x$. Since $\tau$ is discrete, $f_{x}$ is continuous relative to $\tau$; hence, $f_{x}$ is contiguous relative to $F(\tau)$. Since $\{a\}$ and $X-\{a\}$ are both open in $\tau$, there exists a $v \in F(\tau)$ such that $v_{a}=\{a\}$ and $v_{b} \subset X-\{a\}$. Since $f_{x}$ is contiguous, there is a $u \in F(\tau)$ such that $f_{x}\left(u_{x}\right) \subset v_{f_{x}(x)}=v_{a}=a$ and $f_{x}\left(u_{y}\right) \subset v_{f_{x}(y)}=v_{b} \subset X-\{a\}$ for all $y \neq x$. These inclusions imply that $u_{x}=\{x\}$ and $u_{y} \subset X-\{x\}$ for all $y \in X-\{x\}$; whence, the entourage $w$, where $w_{x}=\{x\}$ and $w_{y}=X-\{x\}$ for $y \neq x$, is in $F(\tau)$ because $w$ contains $u$. Since $x$ was an arbitrary point in $X, F(\tau)$ is discrete.

It should be observed that if $(X, C)$ is a space and $A$ is a subset of $X$ which is connected (in $X$ ), then any contiguous image of $A$ is connected (in the image of $X$ ). In particular, the contiguous image of a connected space is connected. Before proceeding to show that functors in $\mathscr{F}$ preserve quasi-components, we give characterizations of quasi-components in terms of continuous and contiguous functions.

THEOREM 2.5. Let $(X, \tau)$ and $(A, \sigma)$ be topological spaces where $A$ has at least two points and $\sigma$ is the discrete topology. Let $\left\{f_{\alpha}: \alpha \in I\right\}$ be the set of continuous functions from $X$ to $A, x \in X$, and $M_{\alpha}=\left\{y: f_{\alpha}(y)=f_{\alpha}(x)\right\}$. Then $Q_{x}$, the quasicomponent of $X$, is the intersection over I of the $M_{\alpha}$ 's.

Proof. We need only to show that $\left\{M_{\alpha}: \alpha \in I\right\}$ exhausts the collection of clopen (closed and open) sets containing $x$. Since $\sigma$ is discrete, each $M_{\alpha}$ is clearly clopen. On the other hand, if $S \subset X$ contains $x$ and is clopen, then the function $f: X \rightarrow A$ such that $f(y)=a \in A$ if $y \in S$ and $f(y)=b \neq a$ if $y \notin S$, is continuous. Thus, $S$ is a member of $\left\{M_{\alpha}: \alpha \in I\right\}$.

THEOREM 2.6. Let $(X, C)$ and $(A, D)$ be spaces such that $A$ has at least two points and $D$ is a discrete continguity. Let $\left\{f_{\alpha}: \alpha \in I\right\}$ be the set of contiguous functions from $X$ to $A, x \in X$, and $M_{\alpha}=\left\{y: f_{\alpha}(y)=f_{\alpha}(x)\right\}$. Then $Q_{x}$, the quasi-component of $x$, is the intersection of all the $M_{\alpha}^{\prime}$ 's.

Proof. Let $u \in C$ and $S$ be the $u$-component of $x$. Then $f: X \rightarrow A$, where $f(y)$ $=a \in A$ if $y \in S$ and $f(y)=b \neq a$ if $y \notin S$, is contiguous; hence, $S \in\left\{M_{\alpha}: \alpha \in I\right\}$ and $\cap M_{\alpha}$ is contained in the intersection over $C$ of the $u$-components of $X$; i.e., $\cap M_{\alpha} \subset Q_{x}$. If $y \notin M_{\alpha}$ for some $\alpha \in I$, then $f_{\alpha}(y) \neq f_{\alpha}(x)$. Let $v \in D$ such that $v_{a}=\{a\}$ and $v_{b}=A-\{a\}$ for all $b \in A$ such that $b \neq a$, where $a=f_{\alpha}(x)$. Let $u \in C$ 
such that $f_{\alpha}\left(u_{z}\right) \subset v_{f_{\alpha}(z)}$ for all $z \in X$. Since the image of the $u$-component of $x$ under $f_{\alpha}$ must be $v$-connected, $f_{\alpha}(z)=a$ for all $z$ in the $u$-component of $x$. Thus, $y$ is not the $u$-component of $x$, and therefore, is not in $Q_{x}$. Thus, $Q_{x} \subset \cap M_{\alpha}$.

THEOREM 2.7. (a) If $(X, C)$ is any space and $x \in X$, then the quasi-component of $x$ relative to $T(C)$ is contained in the quasi-component of $x$ relative to $C$, and (b) if $(X, \tau)$ is any topological space and $x \in X$, then the quasi-component of $x$ relative to $\tau$ is the same as the quasi-component of $x$ relative to $F(\tau)$ for any $F \in \mathscr{F}$.

Proof. (a) Let $(A, D)$ be a discrete space. Then $T(D)$ is the discrete topology for $A$. Since $T$ is a functor, the collection of functions which are contiguous relative to $C$ and $D$ is contained in the collection of functions which are contiguous relative to $T(C)$ and $T(D)$. Thus, by the characterizations of the quasi-components of a point given in Theorems 2.6 and 2.7, the quasi-component of a point $x \in X$ relative to $T(C)$ is contained in the quasi-component of $x$ relative to $C$. (b) By part (a), the quasi-component of a point $x$ relative to $\tau=T F(\tau)$ is contained in the quasicomponent of $x$ relative to $F(\tau)$. By an argument which is similar to the proof of part (a), the quasi-component of $x$ relative to $F(\tau)$ is contained in the quasicomponent of $x$ relative to $\tau$.

Corollary. (a) If $(X, C)$ is a space and $X$ is connected relative to the topology $T(C)$, then $X$ is connected relative to the contiguity $C$, and $(b)$ if $(X, \tau)$ is a topological space and $F \in \mathscr{F}$, then $X$ is connected relative to $F(\tau)$ iff $X$ is connected relative to $\tau$.

3. Coherently connected spaces. Let $(X, C)$ be a space, $u \in C$, and $x, y \in X$. A $u$-edge path from $x$ to $y$ was defined in $\S 1$. Two $u$-edge paths $a=a(1), \ldots, a(n)$ and $b=b(1), \ldots, b(m)$ from $x$ to $y$ are simply related provided that one of the following conditions holds:

(1) $\boldsymbol{a}=\boldsymbol{b}$.

(2) there is an integer $j$ such that $u_{b(j-1)} \cap u_{b(j)} \cap u_{b(j+1)} \neq \varnothing$ and $b(1), \ldots$, $b(j-1), b(j+1), \ldots, b(m)=a$.

(3) $b$ can be obtained from $\boldsymbol{a}$ in the manner of (2).

$\boldsymbol{a}$ and $\boldsymbol{b}$ are equivalent provided there exists a finite sequence $c^{1}, c^{2}, \ldots, c^{p}$ of $u$-edge paths, each from $x$ to $y$, such that $c^{i}$ and $c^{i+1}$ are simply related for $i=1, \ldots$, $p-1$. We shall denote such an equivalence by " $\boldsymbol{a} \sim \boldsymbol{b}(\bmod u)$ " or by simply " $\boldsymbol{a} \sim \boldsymbol{b}$ " when there is no danger of confusion. If $u, v \in C$ with $u \subset v$ then any $u$-edge path is a priori a $v$-edge path. An edge path from $x$ to $y$ is a collection $\left\{a_{u}: u \in C\right\}$ where $a_{u}$ is a $u$-edge path from $x$ to $y$ and where if $u, v \in C$ with $u \subset v$ then $a_{u} \sim a_{v}(\bmod v)$. The space $(X, C)$ is coherently connected (or edge path connected) provided that for each pair $x, y \in X$ there is an edge path from $x$ to $y$. A coherently connected space is connected but the converse is false. (Examples 5.4, 5.5.)

Lemma 3.1. Let $(X, C)$ and $(Y, D)$ be spaces, $f: X \rightarrow Y$ contiguous, $v \in D$, $u \in C$ such that $f\left(u_{x}\right) \subset v_{f(x)}$ for all $x \in X$. If $r, s \in X$ and $a=a(1), \ldots, a(n)$ and 
$\boldsymbol{b}=b(1), \ldots, b(m)$ are $u$-edge paths from $\boldsymbol{r}$ to $s$ with $\boldsymbol{a} \sim \boldsymbol{b}$, then $f(\boldsymbol{a}) \sim f(\boldsymbol{b})(\bmod v)$ where, for example, $f(a)=f(a(1)), \ldots, f(a(n))$.

Proof. Clearly $f(a)$ and $f(b)$ are $v$-edge paths from $f(r)$ to $f(s)$ and if $c^{1}$ and $c^{2}$ are simply related, then $f\left(c^{1}\right)$ and $f\left(c^{2}\right)$ are simply related.

THEOREM 3.2. The contiguous image of a coherently connected space is coherently connected.

Proof. Let $f: X \rightarrow Y$ be contiguous and onto with contig $X=C$, contig $Y=D$. Let $r, s \in X,\left\{a_{u}: u \in C\right\}$ be an edge path from $r$ to $s$ and $\alpha: D \rightarrow C$ be a function such that $f\left(\alpha(v)_{x}\right) \subset v_{f(x)}$ for all $x \in X$ and for all $v \in D$. If $v, w \in D$ with $w \subset v$, then $\boldsymbol{a}_{\alpha(v)} \sim \boldsymbol{a}_{\alpha(v) \cap \alpha(w)}(\bmod \alpha(v))$ and $\boldsymbol{a}_{\alpha(w)} \sim \boldsymbol{a}_{\alpha(v) \cap \alpha(w)}(\bmod \alpha(w))$. By Lemma 3.1, $f\left(a_{\alpha(v)}\right)$ $\sim f\left(a_{\alpha(v) \cap \alpha(w)}\right)(\bmod v)$ and $f\left(a_{\alpha(w)}\right) \sim f\left(a_{\alpha(v) \cap \alpha(w)}\right)(\bmod w)$. The latter equivalence implies $f\left(a_{\alpha(w)}\right) \sim f\left(a_{\alpha(v) \cap \alpha(w)}\right)(\bmod v)$ and, by transitivity, $f\left(a_{\alpha(w)}\right) \sim f\left(a_{\alpha(v)}\right)(\bmod v)$. Thus $\left\{f\left(a_{\alpha(v)}\right): v \in D\right\}$ is an edge path from $f(r)$ to $f(s)$.

Let $(X, C)$ be a space, $u \in C, x, y, z \in X$, and $a=a(1), \ldots, a(n)$ and $b=b(1), \ldots$, $b(m)$ be $u$-edge paths from $x$ to $y$ and $y$ to $z$, respectively. Then $a * b=a(1), \ldots$, $a(n), b(2), \ldots, b(m)$ is a $u$-edge path from $x$ to $z$. Moreover, if $c$ and $d$ are also $u$-edge paths from $x$ to $y$ and $y$ to $z$, respectively, such that $a \sim c, b \sim d$, then $a * b$ $\sim \boldsymbol{c} * \boldsymbol{d}$. Thus, if $\left\{\boldsymbol{a}_{u}\right\}$ and $\left\{\boldsymbol{b}_{u}\right\}$ are edge paths from $x$ to $y$ and $y$ to $z$, respectively, then $\left\{\boldsymbol{a}_{u}\right\} *\left\{\boldsymbol{b}_{u}\right\}=\left\{\boldsymbol{a}_{u} * \boldsymbol{b}_{u}\right\}$ is an edge path from $x$ to $z$.

Let $u, v \in C$ and let $a=a(1), \ldots, a(n)$ and $b=b(1), \ldots, b(m)$ be $u$ - and $v$-edge paths, respectively from $x$ to $y . b$ is partitioned in $a$ provided there is a subsequence $b\left(j_{1}\right), \ldots, b\left(j_{n+1}\right)$ of $\boldsymbol{b}$ such that:

(1) $b\left(j_{1}\right)=b(1)=x$ and $b\left(j_{n+1}\right)=b(m)=y$.

(2) $b\left(j_{k}\right) \in u_{a(k-1)} \cap u_{a(k)}, k=2, \ldots, n$.

(3) $\boldsymbol{b}^{k}=b\left(j_{k}\right), b\left(j_{k}+1\right), \ldots, b\left(j_{k+1}\right)$ is a $v$-edge path in the subspace $u_{a(k)}$ for $k=1, \ldots, n$.

Note that $b=b^{1} * b^{2} * \cdots * b^{n}$.

Lemma 3.3. Let $(X, C)$ be a space, $x, y \in X, u \in C$, and $a$ and $b$ be u-edge paths from $x$ to $y$. If $b$ is partitioned in $a$ then $b \sim a$.

Proof. Let $a=a(1), \ldots, a(n)$ and $b=b(1), \ldots, b(m)$, and $b\left(j_{1}\right), \ldots, b\left(j_{n+1}\right)$ be the subsequence of $b$ satisfying (1), (2), and (3) of the definition above. The following sequence of $u$-edge paths from $x$ to $y$ renders $\boldsymbol{a} \sim \boldsymbol{b}$ :

$$
\begin{gathered}
\boldsymbol{b}= \\
a(1), b(2), \ldots, b\left(j_{2}\right), \ldots, b(m) \\
\\
a(1), b(3), \ldots, b\left(j_{2}\right), \ldots, b(m) \\
\quad \vdots \\
a(1), b\left(j_{2}\right), \ldots, b(m) \\
\\
a(1), a(2), b\left(j_{2}\right), \ldots, b(m) \\
\\
a(1), a(2), b\left(j_{2}+1\right), \ldots, b(m) \\
\quad \vdots \\
a=a(1), a(2), \ldots, a(n) .
\end{gathered}
$$


THEOREM 3.4. If the space $(X, C)$ is connected and locally connected and $C$ has a countable base, then $(X, C)$ is coherently connected.

Proof. Let $B=v(1), v(2), \ldots$ be a countable base for $C$ such that $v(j) \supset v(j+1)$. (If $B^{1}=v_{1}, v_{2}, \ldots$ is any countable base, let $B=v_{1}, v_{1} \cap v_{2}, \ldots$ ) Let $D$ be a base for $C$ such that for each $u \in D, u_{x}$ is a connected subspace for each $x \in X$. Let $u(j) \in D$ such that $u(j) \subset v(j)$ for $j=1,2, \ldots$ Let $x, y \in X$ and $a^{1}$ be a $u(1)$-edge path from $x$ to $y \cdot a^{1}$ is a priori a $v(1)$-edge path. Suppose that a $u(j)$-edge path $a^{j}$ from $x$ to $y$ has been constructed for $j=1, \ldots, n$ such that $\boldsymbol{a}^{j} \sim \boldsymbol{a}^{j-1}(\bmod v(j-1))$ for $j=2, \ldots, n$. Since $u(n)_{x}$ is connected for all $x \in X$, there exists a $u(n+1)$-edge path $\boldsymbol{a}^{n+1}$ from $x$ to $y$ such that $\boldsymbol{a}^{n+1}$ is partitioned in the $u(n)$-edge path $\boldsymbol{a}^{n}$ (although $a^{n+1}$ may not be a $u(n)$-edge path). $a^{n+1}$ is a $v(n+1)$-edge path, hence also a $v(n)$-edge path. Moreover, since $\boldsymbol{a}^{n+1}$ is partitioned in the $v(n)$-edge path $\boldsymbol{a}^{n}$, $\boldsymbol{a}^{n+1} \sim \boldsymbol{a}^{n}(\bmod v(n))$, by Lemma 3.3. Thus there exists a sequence $\boldsymbol{a}^{n}: n=1,2, \ldots$ where $a^{n}$ is a $v(n)$-edge path from $x$ to $y$ and $a^{n} \sim a^{n-1}(\bmod v(n-1))$. If $w \in C$, let $v(j) \in B$ such that $v(j) \subset w$ and let $\boldsymbol{a}^{w}=a^{j}$. Then the collection $\left\{\boldsymbol{a}^{w}: w \in C\right\}$ is an edge path from $x$ to $y$.

THEOREM 3.5. Let $(I, \tau)$ be the unit interval with the usual topology, $F$ a functor in $F$ such that $F(\tau)$ is a pseudo-uniformity for I. If a topological space $(X, \sigma)$ is path connected then $(X, F(\sigma))$ is coherently connected.

Proof. It suffices to show that $(I, P(\tau))$ is coherently connected, where $P(\tau)$ is the largest pseudo-uniformity for $I$ whose derived topology is $\tau$, since by Theorem 3.2, $(I, F(\tau))$ is coherently connected if $(I, P(\tau))$ is, for any pseudo-uniformity $F(\tau) \subset P(\tau)$; and, if $\left\{a_{u}: u \in F(\tau)\right\}$ is an edge path from 0 to 1 in $I, \omega: I \rightarrow X$ is a path then $\omega$ and $\left\{a_{u}: u \in F(\tau)\right\}$ induce an edge path from $\omega(0)$ to $\omega(1)$ in $X$.

Let $u, v \in P(\tau)$ be such that $u_{x}$ and $v_{x}$ are open intervals for each $x \in I$ and such that $u \subset v$. Let $t \in I$ and $\boldsymbol{a}_{u}=a(1), \ldots, a(n), \boldsymbol{b}_{v}=b(1), \ldots, b(m)$ be $u$ - and $v$-edge paths, respectively, from 0 to $t$. A u-edge path is in reduced form if it fails to be a $u$-edge path when any of its points are deleted. If $a^{1}$ is the reduced form of the $u$-edge path $a$ then $a^{1} \sim a$. We assume that $a_{u}$ and $b_{v}$ above, as $v$-edge paths, are in reduced form. This assumption and the notation $\left(a_{i}, a^{i}\right)$ for the interval $v_{a(i)}$, $\left(b_{j}, b^{j}\right)$ for the interval $v_{b(j)}$ imply that

(1) $a_{i}<a_{i+1}$ and $a^{i}<a^{i+1} \quad i=1, \ldots, n-1$.

(2) $b_{j}<b_{j+1}$ and $b^{j}<b^{j+1} \quad j=1, \ldots, m-1$.

Let $k_{i}$ be the smallest integer for which $v_{a\left(k_{i}\right)}$ meets $v_{b(i)}$. For example, $k_{1}=1$, $k_{2}=1$ and in general, $k_{i} \leqq k_{i+1}$. Consider the $v$-edge path $c=a(1), b(1), a(1), b(2)$, $a(2), b(2), \ldots, a\left(j_{3}-1\right), b(2), a\left(j_{3}\right), b(3), a\left(j_{3}+1\right), b(3), \ldots, a\left(j_{4}-1\right), b(3), a\left(j_{4}\right)$, $b(4), \ldots, a\left(j_{m}\right), b(m)$. The conditions (1) and (2) insure that for each triad of successive points of the form $a(j), b(i), a(k)$ or $b(j), a(i), b(k), v_{a(j)} \cap v_{b(i)} \cap v_{a(k)}$ and $v_{b(j)} \cap v_{a(i)} \cap v_{b(k)}$ are not empty. Thus deleting successively each $b(j)$ from $c$ gives a sequence of $v$-edge paths which renders $c$ equivalent to a $v$-edge path which con- 
tains no points of $\boldsymbol{b}_{v}$ and repetitions of points of $\boldsymbol{a}_{u}$. Since the latter $v$-edge path is equivalent to $\boldsymbol{a}_{u}, \boldsymbol{c} \sim \boldsymbol{a}_{u}(\bmod v)$. Similarly $\boldsymbol{c} \sim \boldsymbol{b}_{v}(\bmod v)$, whence $\boldsymbol{a}_{u} \sim \boldsymbol{b}_{v}(\bmod v)$.

Let $B$ be a base for $P(\tau)$ such that for each $u \in B, u_{x}$ is an open interval for each $x \in I$. Let $\left\{a_{u}: u \in B\right\}$ be any collection of finite sequences in $I$ such that $a_{u}$ is a $u$-edge path from 0 to $t$. If $u, v \in B$ and $u \subset v$ then, by the preceding paragraph $\boldsymbol{a}_{u} \sim \boldsymbol{a}_{v}(\bmod v)$. If $w \in P(\tau)$, let $u \in B$ such that $u \subset w$. Define $\boldsymbol{a}_{w}=\boldsymbol{a}_{u}$, thus $\boldsymbol{a}_{w}$ is, in particular, a $w$-edge path from 0 to $t$. The collection $\left\{\boldsymbol{a}_{w}: w \in P(\tau)\right\}$ of sequences formed in this way is an edge path from 0 to $t$. It follows that $(I, P(\tau))$ is coherently connected.

4. The edge path group. Let $(X, C)$ be a space, $x, y \in X$, and $u \in C$. Let $x_{u}$ be the $u$-edge path $\{x\}$ and if $a=a(1), \ldots, a(n)$ is a $u$-edge path from $x$ to $y$, let $a^{-1}=a(n), \ldots, a(1)$. Then $a * a^{-1} \sim x_{u}$ and $a^{-1} * a \sim y_{u}$; moreover, for any $u$-edge path $\boldsymbol{b}$ from $x$ to $y, \boldsymbol{x}_{u} * \boldsymbol{b} \sim \boldsymbol{b}$ and $\boldsymbol{b} * \boldsymbol{y}_{u} \sim \boldsymbol{b}$. If $\boldsymbol{A}=\left\{\boldsymbol{a}_{u}: u \in C\right\}$ and $\boldsymbol{B}=\left\{\boldsymbol{b}_{u}: u \in C\right\}$ are edge paths from $x$ to $y$ then $A$ is equivalent to $B(A \sim B)$ iff $a_{u} \sim b_{u}$ for all $u \in C$. Let $\boldsymbol{x}=\left\{\boldsymbol{x}_{u}: u \in C\right\}$ and $A^{-1}=\left\{a_{u}^{-1}: u \in C\right\}$. Then $\boldsymbol{x} * \boldsymbol{A}=\left\{\boldsymbol{x}_{u} * a_{u}: u \in C\right\}$ $\sim A, A * y \sim A, A * A^{-1} \sim x$, and $A^{-1} * A \sim y$. Let $[A]$ be the equivalence class of the edge path $\boldsymbol{A}$. Define $[\boldsymbol{A}][\boldsymbol{B}]=[\boldsymbol{A} * \boldsymbol{B}]$ whenever $\boldsymbol{A} * \boldsymbol{B}$ is defined. This is a well-defined operation on equivalence classes. The edge path group $E(X, C, x)$ of a pointed space $(X, C, x)$ is the set of equivalence classes of edge loops at $x$ (edge paths beginning and ending at $x$ ) with the operation defined above. The identity of $E(X, C, x)$ is $[x]$ and if $A$ is an edge loop at $x,[A]^{-1}=\left[A^{-1}\right]$.

ThEOREM 4.1. Let $\left(X, C, x_{0}\right)$ and $\left(Y, D, y_{0}\right)$ be pointed spaces, $f:\left(X, x_{0}\right) \rightarrow\left(Y, y_{0}\right)$ be contiguous and $\alpha: D \rightarrow C$ be a function satisfying the property $K: f\left(\alpha(v)_{x}\right) \subset v_{f(x)}$ for all $x \in X$ and all $v \in D$. Then $f^{*}: E\left(X, C, x_{0}\right) \rightarrow E\left(Y, D, y_{0}\right):$ if $A=\left\{a_{u}: u \in C\right\}$ is an edge loop at $x_{0}, f^{*}([A])=\left[\left\{f\left(a_{\alpha(v)}\right): v \in D\right\}\right]$, is a homomorphism which is independent of the choice of a function satisfying condition $K$.

Proof. The existence of a function $\alpha$ satisfying $K$ follows from the hypothesis that $f$ is contiguous. That $\left\{f\left(a_{\alpha(v)}\right): v \in D\right\}$ is an edge loop at $y_{0}$ follows from the proof of Theorem 3.2 and it is straightforward argument to show that $f^{*}$ is a homomorphism. If $\alpha, \beta: D \rightarrow C$ are functions satisfying $K$, let $\gamma(v)=\alpha(v) \cap \beta(v)$ for each $v \in D$. Then $\boldsymbol{a}_{\gamma(v)} \sim \boldsymbol{a}_{\alpha(v)}(\bmod \alpha(v))$ and $\boldsymbol{a}_{\gamma(v)} \sim \boldsymbol{a}_{\beta(v)}(\bmod \beta(v))$, whence $f\left(a_{\alpha(v)}\right) \sim f\left(a_{\gamma(v)}\right) \sim f\left(a_{\beta(v)}\right)(\bmod v)$ and $\left\{f\left(a_{\alpha(v)}\right): v \in D\right\} \sim\left\{f\left(a_{\beta(v)}\right): v \in D\right\}$. Thus the definition of $f^{*}$ does not depend on the choice of a function satisfying $K$.

REMARK. If $f:\left(X, x_{0}\right) \rightarrow\left(Y, y_{0}\right)$ and $g:\left(Y, y_{0}\right) \rightarrow\left(Z, z_{0}\right)$ are contiguous, then $(g \circ f)^{*}=g^{*} \circ f^{*}$, and $l_{X}^{*}=l_{E\left(X, C, x_{0}\right)}$. Thus $E$, where $E$ assigns a pointed space $\left(X, C, x_{0}\right)$ to the group $E\left(X, C, x_{0}\right)$ and a contiguous function $f$ to the homomorphism $f^{*}$, is a covariant functor from the category $\mathscr{S}^{*}$ of pointed spaces to the category $\mathscr{G}$ of groups.

Example 5.4 shows that the group $E\left(X, C, x_{0}\right)$ may depend on the base point $x_{0}$. However, 
THEOREM 4.2. If $(X, C)$ is coherently connected and $x_{0}, x_{1} \in X$, then $E\left(X, C, x_{0}\right)$ is isomorphic to $E\left(X, C, x_{1}\right)$.

Proof. (The proof is analogous to the proof of the independence of $\pi_{1}\left(X, x_{0}\right)$ of the base point when $X$ is a path connected topological space.)

Let $[a]$ be the equivalence class of a $u$-edge loop $a$ at $x_{0}$ where $u$ is an entourage in $C$. The group $E_{u}\left(X, x_{0}\right)$, whose elements are of the form [a] where $a$ is a $u$-edge loop at $x_{0}$ and with the operation $[a] \cdot[b]=[a * b]$ is called the $u$-component of the edge path group $E\left(X, C, x_{0}\right)$.

RemarK. A space $(X, C)$ is an elementary space provided $C$ has a single entourage as a base. Let $(X, C)$ be an arbitrary space and let $X_{u}=\left(X, C_{u}\right)$ be the elementary space for which $C_{u}$ is generated by an entourage $u \in C$. If $u, v \in C$ with $u \subset v$, then $1_{X}: X_{u} \rightarrow X_{v}$ is contiguous. Let $C$ be partially ordered by: $u \geqq v$ iff $u \subset v$. The collection $\left\{\left(X_{u}, 1_{X}\right): u \in C\right\}$ is a projective limit system whose projective limit is $(X, C)$. Thus every space is the projective limit of a collection of elementary spaces. The $u$-component $E_{u}\left(X, x_{0}\right)$ of the edge path group $E\left(X, C, x_{0}\right)$ may also be interpreted as the edge path group of the elementary pointed space $\left(X, C_{u}, x_{0}\right)$. For $u, v \in C$, let $\phi_{u v}: E_{u}\left(X, x_{0}\right) \rightarrow E_{v}\left(X, x_{0}\right)$ be the homomorphism induced by $1_{X}:\left(X_{u}, x_{0}\right) \rightarrow\left(X_{v}, x_{0}\right)$ as in Theorem 4.1. Then $\phi_{u v}([a])=[a]$ where on the right [a] is the $v$-equivalence class of the $v$-edge loop a. $\phi_{u v}$, incidentally, may be neither a monomorphism nor an epimorphism (Examples 5.3 and 5.4). The collection $\left\{\left(E_{u}\left(X, x_{0}\right), \phi_{u v}\right): u, v \in C, u \subset v\right\}$ where $C$ is partially ordered by inclusion is a projective system of groups.

THEOREM 4.3. The edge path group of a space $\left(X, C, x_{0}\right)$ is isomorphic to $\operatorname{proj} \lim \left\{E_{u}\left(X, x_{0}\right), \phi_{u v}\right\}$.

Proof. $\left\{\left[a_{u}\right]: u \in C\right\}$ is an element of $\operatorname{proj} \lim \left\{E_{u}\left(X, x_{0}\right), \phi_{u v}\right\}$ where $a_{u}$ is a $u$-edge loop at $x_{0}$, iff for each $u, v \in C$ with $u \subset v, \phi_{u v}\left(\left[a_{u}\right]\right)=\left[a_{v}\right]$ iff $\boldsymbol{a}_{u} \sim \boldsymbol{a}_{v}(\bmod v)$ iff $\left\{a_{u}: u \in C\right\}$ is an edge loop at $x_{0}$. Let $\phi: \operatorname{proj} \lim \left\{E_{u}\left(X, x_{0}\right), \phi_{u v}\right\} \rightarrow E\left(X, C, x_{0}\right)$ be defined by $\phi\left(\left\{\left[\boldsymbol{a}_{u}\right]: u \in C\right\}\right)=\left[\left\{\boldsymbol{a}_{u}: u \in C\right\}\right]$. Then $\phi$ is an isomorphism.

Let $\left(X, C, x_{0}\right)$ and $\left(Y, D, y_{0}\right)$ be spaces, $f:\left(X, x_{0}\right) \rightarrow\left(Y, y_{0}\right)$ be contiguous, and let $\alpha: D \rightarrow C$ be a function satisfying condition $K$ of Theorem 4.1. Then $f^{* *}: \operatorname{proj} \lim \left\{E_{u}\left(X, x_{0}\right), \phi_{u v}\right\} \rightarrow$ proj $\lim \left\{E_{w}\left(Y, y_{0}\right), \phi_{w t}\right\}$, such that

$$
f^{* *}\left(\left\{\left[a_{u}\right]: u \in C\right\}\right)=\left\{\left[f\left(a_{\alpha(w)}\right)\right]: w \in D\right\},
$$

is a homomorphism. The isomorphism $\phi$ of Theorem 4.3 is natural in the sense that the diagram

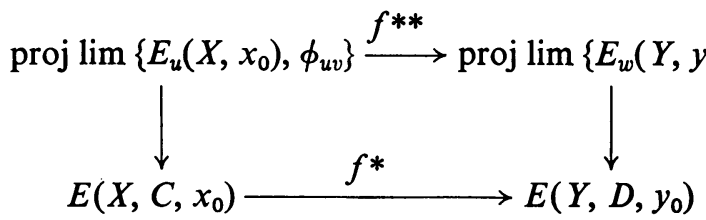

is commutative. 
5. Examples. The usual uniformity $C$ for the plane $R^{2}$ is generated by the base $\{u(n): n=1, \ldots\}$ of entourages where for each $x \in R^{2}, u(n)_{x}=\{y: d(x, y)<1 / n\}$. Subspaces of $\left(R^{2}, C\right)$ are used in several of the examples which follow.

EXAMPLE 5.1. Let $Q^{2}$ be the subspace of $\left(R^{2}, C\right)$ consisting of all points whose coordinates are rational. Then $Q^{2}$ is connected, locally connected, coherently connected, and $E\left(Q^{2}, p\right)=1$ for any $p \in Q^{2}$.

EXAMPLE 5.2. Let $S$ be the subset of $R^{2}$ consisting of the unit circle with the point $(1,0)$ deleted. If $C_{1}$ is the relative uniformity, then, since none of the groups $E_{u(n)}(S, p)$ "see" the hole in $S, E_{u(n)}(S, p)=Z, n=1, \ldots$, and $E\left(S, C_{1}, p\right)=Z$. On the other hand, let $\tau$ be the topology for $X$ inherited from the usual topology of the plane. Let $v$ be the entourage in $S$ such that $v_{x}=w_{x} \cap S$ where $w_{x}$ is a neighborhood of $x$ in $R^{2}$ which misses $(1,0)$. The collection of entourages in the pseudo-uniformity $P(\tau)$ (Example 2.2) which are contained in $v$ is a base for $P(\tau)$, and for each such entourage $u, E_{u}(S, p)=1$. Thus $E(S, P(\tau), p)=1$. Hence, if $C_{1}$ and $C_{2}$ are different contiguities derived from the same topology, $E\left(X, C_{1}, p\right)$ and $E\left(X, C_{2}, p\right)$ are not generally the same.

EXAMPLE 5.3. For each positive integer $n$, let $S(n, 1 / n)$ be the open disc about $(n, 0)$ with radius $1 / n$. Let $S=R^{2}-\bigcup_{n=1}^{\infty} S(n, 1 / n)$ and let $C_{1}$ be the relative uniformity for $S$ inherited from $\left(R^{2}, C\right)$. Each group $E_{u(n)}(S, p)$ fails to "see" a hole of radius less than $1 / n$. Thus if $m>n$, the canonical homomorphism

$$
\phi_{u(m) u(n)}: E_{u(m)}(S, p) \rightarrow E_{u(n)}(S, p)
$$

is not a monomorphism. $E_{u(n)}(S, p)$ is finitely generated but $E\left(S, C_{1}, p\right)$ is not.

EXAMPLE 5.4. Let $L_{1}=\left\{(x, y) \in R^{2}: y=0, x \geqq 0\right\}, L_{2}=\left\{(x, y) \in R^{2}: y=1, x \geqq 0\right\}$, $S_{n}=\left\{(x, y) \in R^{2}: x=n, 0 \leqq y \leqq 1\right\}-\{(x, y): x=n, 0<y<1 /(n+1)\}, n=0,1,2, \ldots$, $K=\{(x, y): y=3 / 4,1 \leqq x \leqq 2\}$ and let $S=L_{1} \cup L_{2} \cup\left(\cup_{n=0}^{\infty} S_{n}\right) \cup K$ (see diagram).

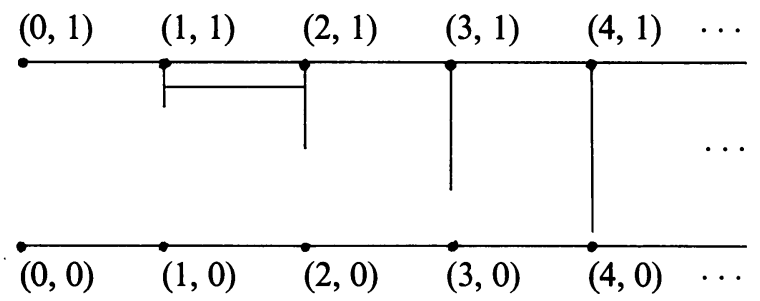

Let $S$ have the relative uniformity $C_{1}$ inherited from $\left(R^{2}, C\right)$. For each positive integer $m$, there is a $u(m)$-edge path from $(0,0)$ to $(0,1)$ through the segment $S_{m}$; hence $S$ is connected. $S$ is not locally connected. Let $a_{u(n)}$ be a $u(n)$-edge path from $(0,0)$ to $(0,1)$ for $n=1,2,3, \ldots$ For each $n, a_{u(n)}$ is through a segment $S_{m}$ where $m \geqq n$. Let $k=m+1$. Then $a_{u(k)}$ is through a segment $S_{p}$ where $p \geqq k$ and, in particular, $k \neq m$. Thus, $u(k) \subset u(n)$ but $\boldsymbol{a}_{u(k)}$ is not equivalent to $\boldsymbol{a}_{u(n)}(\bmod u(n))$. Therefore, $S$ cannot be coherently connected.

Each group $E_{u(n)}(S, x)$, for any $x \in S$, has infinitely many generators; although if $x \in L_{1}, E\left(S, C_{1}, x\right)$ is trivial and if $x \in S-L_{1}, E\left(S, C_{1}, x\right)=Z$. Thus, if $(X, C)$ is 
not coherently connected, the edge path group depends on the base point. Moreover, this example also shows that the canonical homomorphisms $\phi_{u(m) u(n)}, m>n$, need not be epimorphisms.

EXAMPLE 5.5. Let $S$ be the graph of $y=\sin 1 / x, 0<x \leqq 1$ and the point $(0,0)$ and let $\tau$ be the relative topology for $S$. Let

and

$$
S_{0}=\{(x, y) \in S: 2 / \pi<x \leqq 1\}
$$

$$
S_{k}=\left\{(x, y) \in S: \frac{2}{(2 k+1) \pi}<x \leqq \frac{2}{(2 k-1) \pi}\right\} \text { for } k=1,2,3, \ldots
$$

Let $u$ be an entourage in $S$ such that $u_{(0,0)}$ is the intersection of $S$ with the rectangle $\{(x, y):|x|<1 / 4,|y|<1 / 4\}$ and if $p \in S_{k}$ then $u_{p}$ is the intersection of $S$ with an open disc $d_{p}$ about $p$ where $d_{p}$ meets neither $S_{k+1}$ nor $S_{k-1}$ if $p$ is an interior point of $S_{k}$, and $d_{p}$ has radius less than $1 / 8$ and does not meet $S_{k+1}$ if $p=(2 /(2 k-1) \pi \pm 1)$. Let $a_{u}=a(1), \ldots, a(n)$ be a $u$-edge path from $(0,0)$ to a point $p \in S, p \neq(0,0)$. Assume $a(2) \in S_{k}$. Let $v$ be the entourage in $S$ for which $v_{x}=u_{x}$ if $x \neq(0,0)$ and $v_{(0,0)}$ is the intersection of $S$ with the open rectangle $\left\{(x, y) \in R^{2}: x<2 /(2 k+3) \pi\right.$, $y<1 / 4\}$. Then $v$ and $u$ are entourages in the pseudo-uniformity $P(\tau)$ and $v \subset u$. If $\boldsymbol{a}_{v}$ is any $v$-edge path from $(0,0)$ to $p$ then $\boldsymbol{a}_{v}$ must pass through $S_{k+1}$, and hence cannot be equivalent to $a_{u}(\bmod u)$. Therefore $(S, P(\tau))$ is not coherently connected.

The collection of entourages in $P(\tau)$ which are contained in $u$ is a base for $P(\tau)$. For each such entourage $w, E_{w}(S, p)$ has infinitely many generators for any $p \in S$, although $E(S, P(\tau), p)$ is trivial.

6. The edge path group as a topological invariant. Let $E_{F}$ be the composition of $E$ with a functor $F \in \mathscr{F}(\S 2)$. Then for each $F \in \mathscr{F}, E_{F}$ is a functor from the category of pointed topological spaces to the category of groups.

THEOREM 6.1. Let $C_{1}$ and $C_{2}$ be contiguities for $X$ such that $C_{1} \subset C_{2}$. There exists an epimorphism $\psi: E\left(X, C_{2}, x\right) \rightarrow E\left(X, C_{1}, x\right)$ where $x \in X$.

Proof. The homomorphism $\psi$ is derived from the inclusion function $i$ : $C_{1} \rightarrow C_{2}$ and Theorem 4.3.

COROLlaRY. If $F$ is a functor in $\mathscr{F}$ such that $F(\tau)$ is a pseudo-uniformity for $X$ for any topological space $(X, \tau)$, then there is a natural transformation from the functor $E_{P}$ to $E_{F}$ where $P$ is the functor of Example 2.2.

If $u$ is an entourage in $X$, then $\left\{u_{x}: x \in X\right\}$ is a cover of $X$ which will also be denoted by $u$. If $u$ and $v$ are entourages in $X$ such that $u \subset v$, then $u$ refines $v$, but the converse is not true. A contiguity $C$ for $X$ may also be ordered by refinement and by the preceding statement, the inclusion order relation on $C$ is properly contained in the refinement order relation on $C$. If $u$ and $v$ are entourages in $X$ such that $u$ refines $v$, a projection of $u$ onto $v$ is a function $p:\left\{u_{x}: x \in X\right\} \rightarrow$ $\left\{v_{x}: x \in X\right\}$ such that $u_{x} \subset p\left(u_{x}\right)$ for every $x \in X$. If $a=a(1), \ldots, a(n)$ is a sequence 
of points in $X$ and $p: u \rightarrow v$ is a projection, let $p(a)=b(1), \ldots, b(n)$ where $p\left(u_{a(i)}\right)$ $=v_{b(i)}, i=1, \ldots, n$.

TheOREM 6.2. Let $(X, C, x)$ be a pointed space and $u, v \in C$ such that $u$ refines $v$. If $p: u \rightarrow v$ is a projection, then $p^{*}: E_{u}(X, x) \rightarrow E_{v}(X, x): p^{*}([a])=[x, p(a), x]$ $=[x, p(a(1)), \ldots, p(a(n)), x]$ for each u-edge loop a at $x$. Moreover, if $q: u \rightarrow v$ is also a projection, $q^{*}=p^{*}$.

Proof. Since $u_{x} \cap u_{y} \neq \varnothing$ implies $p\left(u_{x}\right) \cap p\left(u_{y}\right) \neq \varnothing, p(a)$ is a $v$-edge path, although not necessarily a $v$-edge loop at $x$. Since $x \in u_{x} \subset p\left(u_{x}\right), x, p(a), x$ is a $v$-edge loop at $x$; thus, $p^{*}([\boldsymbol{a}]) \in E_{v}(X, x)$. If $\boldsymbol{a}$ and $\boldsymbol{b}$ are simply related $u$-edge loops at $x$, then $x, p(a), x$ and $x, p(b), x$ are simply related $v$-edge loops. It follows that $p^{*}$ is a function. Since $x, p(a), p(b), x \sim x, p(a), x, x, p(b), x(\bmod v), p^{*}$ is a homomorphism.

Let $q: u \rightarrow v$ be a projection also, and let $a=a(1), \ldots, a(n)$ be a $u$-edge loop at $x$. The sequence of $v$-edge loops

$$
\begin{aligned}
& x, p(a(1)), p(a(2)), \ldots, p(a(n)), x \\
& x, q(a(1)), p(a(1)), p(a(2)), \ldots, p(a(n)), x \\
& x, q(a(1)), p(a(2)), \ldots, p(a(n)), x \\
& \vdots \\
& x, q(a(1)), \ldots, q(a(n)), p(a(n)), x \\
& x, q(a(1)), \ldots, q(a(n)), x
\end{aligned}
$$

renders $x, p(a), x \sim x, q(a), x(\bmod v)$. It follows from this that $q^{*}([a])=p^{*}([a])$.

THeOREM 6.3. Let $(X, C, x)$ be a pointed space and let $C$ be partially ordered by refinement. The collection $\left\{\left(E_{u}(X, x), p_{u v}\right): u, v \in C, u\right.$ refines $v$ and $p_{u v}: u \rightarrow v$ is $a$ projection $\}$ is a projective system over $C$ whose projective limit $E^{r}(X, C, x)$ is naturally isomorphic to $E(X, C, x)$.

Proof. The collection described in the theorem is clearly a projective system. If $C^{r}$ denotes the contiguity $C$ ordered by refinement, then $j: C \rightarrow C^{r}: u \rightarrow u$ and 1: $E_{j(u)}(X, x)=E_{u}(X, x) \rightarrow E_{u}(X, x)$ constitute a map $\omega:\left\{\left(E_{u}(X, x), P_{u v}\right): u, v \in C^{r}\right\}$ $\rightarrow\left\{\left(E_{u}(X, x), \phi_{u v}\right): u, v \in C\right\}$ which in turn induces an isomorphism between the projective limits of these systems.

COROLlaRY 1. The restrictions of the functions $E_{P}$ and $E_{Q}$ (see Examples 2.2 and 2.3) to compact, pointed topological spaces are naturally equivalent.

Proof. Let $(X, \tau, x)$ be a compact, pointed topological space. By Theorem 6.3, we need only show that $Q(\tau)$ ordered by refinement is cofinal in $P(\tau)$ ordered by refinement. Let $u$ be an entourage in the pseudo-uniformity $P(\tau)$ and let $v \in P(\tau)$ such that $v \subset u$ and $v$ is open relative to $P(\tau) .\left\{v_{x}: x \in X\right\}$ is an open cover of $X$. Let $\left\{v_{x(i)}: i=1, \ldots, n\right\}$ be a finite subcover and let $v_{i}$ be the entourage in the quasi- 
uniformity $Q(\tau)$ derived from the open set $v_{x(i)}, i=1, \ldots, n$. Then $w=\bigcap_{i=1}^{n} v_{i}$ is an entourage in $Q(\tau)$ which refines $v$, and hence $u$.

COROLlaRY 2. The restrictions of the functors $E_{P}, E_{Q}$, and $E_{U}$ (Example 2.5) to compact, pointed, metric spaces are naturally equivalent.

Proof. Let $(X, \tau, x)$ be a compact, pointed, metric space. We need only show that $U(\tau)$ ordered by refinement is cofinal in $P(\tau)$ ordered by refinement. Since $(X, \tau)$ is compact, $U(\tau)$ consists of all neighborhood (relative to the product topology for $X \times X$ ) of the diagonal (Kelley [5, Theorem 29, p. 197]). Let $v$ be an entourage in $P(\tau)$ such that $v$ is open relative to $P(\tau)$. Then $\left\{v_{x}: x \in X\right\}$ is an open cover of $X$. Let $\delta$ be the Lebesgue number of this cover and $w$ be the entourage in $X$ for which $w_{x}=\{y \in X: d(x, y)<\delta\}$. Then $w$ is a neighborhood of the diagonal of $X \times X$ and hence is in $U(\tau)$. Moreover, $w$ refines $v$; whence, $U(\tau)$ is cofinal in $P(\tau)$.

Let $\pi_{1}(X, x)$ be the usual fundamental group of the pointed topological space $(X, \tau, x)$.

THEOREM 6.4. Let $F: \mathscr{T} \rightarrow \mathscr{S}$ be a functor in $\mathscr{F}$ whose object function is into the subcategory of pseudo-uniform spaces. There exists a natural transformation from the functor $\pi_{1}$ to the functor $E_{F}$.

Proof. Let $\sigma$ be the usual topology for the unit interval $I$. $(I, F(\sigma))$ is coherently connected by Theorem 3.5. Let $\left\{a_{u}: u \in F(\sigma)\right\}$ be an edge path from 0 to 1 and $\omega:(I, I) \rightarrow(X, x)$ be continuous where $(X, \tau, x)$ is a pointed topological space. Then $\omega$ is contiguous relative to $F(\sigma)$ and $F(\tau)$. Let $\alpha: F(\tau) \rightarrow F(\sigma)$ be a function such that $\omega\left(\alpha(v)_{t}\right) \subset v_{\omega(t)}$ for all $t \in I$ and $v \in F(\tau)$. Define $\psi: \pi_{1}(X, x) \rightarrow E(X, F(\tau), x)$ such that $\psi([\omega])=\left[\left\{\omega\left(a_{\alpha(v)}\right): v \in F(\tau)\right\}\right]$. If $\beta: F(\tau) \rightarrow F(\sigma)$ is also a function for which $\omega\left(\beta(v)_{t}\right) \subset v_{\omega(t)}$ for all $t \in I, v \in F(\tau)$, then $\left\{\omega\left(\boldsymbol{a}_{\beta(v)}\right): v \in F(\tau)\right\}$ and $\left\{\omega\left(\boldsymbol{a}_{\alpha(v)}\right)\right.$ : $v \in F(\tau)\}$ are equivalent edge loops at $x$. (See the proof of Theorem 4.1.) Thus, the definition of $\psi$ does not depend on the choice of the function $\alpha$. If $\left\{\boldsymbol{b}_{u}: u \in F(\sigma)\right\}$ is also an edge path in $I$ from 0 to 1 , then, from the proof of Theorem 3.5, $\left\{\boldsymbol{b}_{u}: u \in F(\sigma)\right\} \sim\left\{\boldsymbol{a}_{u}: u \in F(\sigma)\right\}$ which implies that $\left\{\omega\left(\boldsymbol{a}_{\alpha(v)}\right): v \in F(\tau)\right\} \sim$ $\left\{\omega\left(\boldsymbol{b}_{\alpha(v)}\right): v \in F(\tau)\right\}$ (Lemma 3.1). Thus, the definition of $\psi$ does not depend on the choice of edge path in $I$ from 0 to 1 .

Let $\gamma:(I, \dot{I}) \rightarrow(X, x)$ be continuous and homotopic, relative to $I$, to $\omega$. Let $h: I \times I \rightarrow X$ be the continuous function effecting this homotopy. If $\sigma^{*}$ is the product topology for $I \times I$, then $h$ is contiguous relative to $F\left(\sigma^{*}\right)$ and $F(\tau)$. Let $v \in F(\tau)$ and $w$ be an open entourage in $F\left(\sigma^{*}\right)$ such that (1) $h\left(w_{p}\right) \subset v_{h(p)}$ for all $p \in I \times I$, and (2) $w \subset u^{*}$ where $u^{*}$ is the entourage in the product contiguity (which is contained in contiguity $\left.F\left(\sigma^{*}\right)\right)$ satisfying $\left(u^{*}\right)_{(s, t)}=u_{s} \times u_{t}$ where $u \in F(\sigma)$ and $\omega\left(u_{s}\right) \subset$ $v_{\omega(s)}, \gamma\left(u_{s}\right) \subset v_{\gamma(s)}$ for all $s, t \in I$. For each positive integer $n$, let $\mathscr{U}(n)=\left\{u_{i j}(n): i\right.$, $\left.j=0,1, \ldots, 2^{n}\right\}$ be the cover of $I \times I$ consisting of the rectangles

$$
u_{i j}(n)=(I \times I) \cap\left\{(s, t):\left|s-i / 2^{n}\right| \leqq 1 / 2^{n},\left|t-j / 2^{n}\right| \leqq 1 / 2^{n}\right\} .
$$


Then the diameter of $u_{i j}(n)$ is less than or equal to $\sqrt{ } 2 / 2^{n-1}$. Let $\delta$ be the Lebesgue number of the open cover $\left\{w_{p}: p \in I \times I\right\}$ and choose $n$ so that $\sqrt{ } 2 / 2^{n-1}<\delta$. Then $\mathscr{U}(n)$ refines the cover $w$. For each pair of integers $(i, j)$ let $(s(i), t(j)) \in I \times I$ be such that $u_{i j}(n) \subset w_{(s(i), t(j))}$. Let $a_{j}$ be the sequence $(0, t(j)),(s(0), t(j)), \ldots$, $\left(s\left(2^{n}\right), t(j)\right),(1, t(j))$. For each $j=0, \ldots, 2^{n}, a_{j}$ is a $w$-edge path since, for example, $(0, t(j)) \in w_{(0, t(j))} \cap w_{(s(0), t(j))}$ and $(s(i), t(j)) \in w_{(s(i), t(j))} \cap w_{(s(i+1), t(j))}$. Moreover, $h\left(a_{j}\right)$ is a $v$-edge loop at $x$. The sequence of $v$-edge loops

$$
\begin{aligned}
& x, h(s(0), t(j)), h(s(1), t(j)), \ldots, h\left(s\left(2^{n}\right), t(j)\right), x \\
& x, h(s(0), t(j+1)), h(s(0), t(j)), \ldots, x \\
& x, h(s(0), t(j+1)), h(s(1), t(j)), \ldots, x \\
& x, h(s(0), t(j+1)), h(s(1), t(j+1)), h(s(1), t(j)), \ldots, x \\
& x, h(s(0), t(j+1)), h(s(1), t(j+1)), h(s(2), t(j)), \ldots, x \\
& \quad \vdots \\
& x, h(s(0), t(j+1)), \ldots, h\left(s\left(2^{n}\right), t(j+1)\right) x
\end{aligned}
$$

renders $h\left(a_{j}\right) \sim h\left(a_{j+1}\right)(\bmod v)$, since for example

$$
\begin{aligned}
h(s(i), t(j)) \in h\left(w_{(s(i), t(j+1))}\right) & \cap h\left(w_{(s(i+1), t(j+1)))}\right) \\
& \cap h\left(w_{(s(i+1), t(j))}\right) .
\end{aligned}
$$

Thus, by transitivity, $h\left(a_{0}\right) \sim h\left(a_{k}\right)(\bmod v)$ where $k=2^{n}$. The sequence $(0,0)$, $(s(0), 0), \ldots,\left(s\left(2^{n}\right), 0\right),(1,0)=b$ is a $u$-edge path in $I$, since it is a $w$-edge path in $I \times I$ and $w \subset u^{*}$. By the proof of Theorem 3.5, $\boldsymbol{b} \sim \boldsymbol{a}_{u}(\bmod u)$; hence $h(\boldsymbol{b})=\omega(\boldsymbol{b}) \sim$ $\omega\left(a_{u}\right)(\bmod v)$. Moreover, the sequence of $v$-edge loops

$$
\begin{gathered}
h(b)=x, h(s(0), 0), h(s(1), 0), \ldots, h\left(s\left(2^{n}\right), 0\right), x \\
x, h(s(0), t(0)), h(s(0), 0), h(s(1), 0), \ldots, x \\
x, h(s(0), t(0)), h(s(1), 0), h(s(2), 0), \ldots, x \\
\quad \vdots \\
h\left(a_{0}\right)=x, h(s(0), t(0)), h(s(1), t(0)), \ldots, h\left(s\left(2^{n}\right), t(0)\right), x
\end{gathered}
$$

renders $h(b) \sim h\left(a_{0}\right)(\bmod v)$; and, by transitivity, $\omega\left(\boldsymbol{a}_{u}\right) \sim h\left(\boldsymbol{a}_{k}\right)$ where $k=2^{n}$. Similarly, $h\left(a_{k}\right) \sim \gamma\left(a_{u}\right)$, and so, finally, $\omega\left(\boldsymbol{a}_{u}\right) \sim \gamma\left(\boldsymbol{a}_{u}\right)$. Thus $\gamma$ homotopic to $\omega$ implies that $\left[\left\{\gamma\left(a_{\alpha(v)}\right): v \in F(\tau)\right\}\right]=\left[\left\{\omega\left(a_{\alpha(v)}\right): v \in F(\tau)\right\}\right]$ and, so, $\psi$ is well defined. $\psi$ is clearly a homomorphism.

TheOrem 6.5. Let $(X, \tau)$ and $(Y, \sigma)$ be topological spaces, $f, g: X \rightarrow Y$ be continuous, homotopic functions, $x_{0} \in X, y_{1}, y_{2} \in Y$ such that $y_{1}=f\left(x_{0}\right), y_{2}=g\left(x_{0}\right)$. Then there is an isomorphism $\psi: E\left(Y, P(\sigma), y_{2}\right) \rightarrow E\left(Y, P(\sigma), y_{1}\right)$ such that $f^{*}=\psi \circ g^{*}$ where, for example, $f^{*}: E\left(X, P(\tau), x_{0}\right) \rightarrow E\left(Y, P(\sigma), y_{1}\right)$.

Proof. Let $H: X \times I \rightarrow Y$ be continuous and such that $H(x, 0)=f(x), H(x, 1)$ $=g(x) . H$ is contiguous relative to the pseudo-uniformities $P\left(\tau^{*}\right)$ and $P(\sigma)$ where $\tau^{*}$ is the product topology. Let $\beta: P(\sigma) \rightarrow P\left(\tau^{*}\right)$ be a function such that $\beta(v)$ is 
open relative to $P\left(\tau^{*}\right)$ and $H\left(\beta(v)_{p}\right) \subset v_{H(p)}$ for all $p \in X \times I$ and all $v \in P(\sigma)$. Let $\left\{b_{u}: u \in P(\rho)\right\}$ be an edge path in $I$ from 0 to 1 where $\rho$ is the usual topology for $I$. The injection $e: I \rightarrow X \times I: e(t)=\left(x_{0}, t\right)$ is continuous, hence contiguous relative to $P(\rho)$ and $P\left(\tau^{*}\right)$. Let $\gamma: P\left(\tau^{*}\right) \rightarrow P(\rho)$ be a function such that $\gamma(w)$ is an open entourage and $e\left(\gamma(w)_{t}\right) \subset w_{e(t)}$ for all $t \in I$ and all $w \in P\left(\tau^{*}\right)$. Let $\psi$ be the isomorphism generated by the edge path $\left\{H e\left(b_{\gamma \beta(v)}\right): v \in P(\sigma)\right\}$ from $y_{1}$ to $y_{2}$. If $\left\{a_{u}: u \in P(\tau)\right\}$ is an edge loop at $x_{0}$ in $X$ and $\alpha: P(\sigma) \rightarrow P(\tau)$ is a function such that $f\left(\alpha(v)_{x}\right) \subset v_{f(x)}$ and $g\left(\alpha(v)_{x}\right) \subset v_{g(x)}$ for all $x \in X$ and $v \in P(\sigma)$, then we need only show that $f\left(a_{\alpha(v)}\right) \sim H e\left(b_{\gamma \beta(v)}\right) * g\left(a_{\alpha(v)}\right) * H e\left(b_{\gamma \beta(v)}\right)^{-1}(\bmod v)$ for each $v \in P(\sigma)$.

Let $v \in P(\sigma)$. Since $\left\{\beta(v)_{p}: p \in X \times I\right\}$ is an open (relative to $\tau^{*}$ ) cover of $X \times I$ there is an open cover $\mathscr{U}$ of $X$ and an open cover $\Omega$ of $X \times I$ stacked over $\mathscr{U}$ which refines the cover $\beta(v)$ (Eilenberg and Steenrod [3, p. 241, Lemma 5.6]). $\Omega$ has the form $\left\{\delta \times \omega(\delta, i): \delta \in \mathscr{U}, i=1, \ldots, n_{\delta}\right\}$ where $\left\{\omega(\delta, i): i=1, \ldots, n_{\delta}\right\}$ is an open cover of $I$. Let $w$ be an entourage in $X$ formed from $\mathscr{U}$ by taking $w_{x}$ to be an element of $\mathscr{U}$ which contains $x$ for each $x \in X$. Then $w$, and hence $u=w \cap \alpha(v)$, is a member of $P(\tau)$; moreover, with reference to the edge loop $\left\{a_{u}: u \in P(\tau)\right\}, a_{u} \sim a_{w}(\bmod w)$ and $\boldsymbol{a}_{u} \sim \boldsymbol{a}_{\alpha(v)}(\bmod \alpha(v))$. Let $\boldsymbol{a}_{u}=a(1), \ldots, a(k)$ and $\omega_{j}=\left\{\omega\left(w_{a(j)}, i\right): i=1, \ldots, n_{j}\right\}$ where $\left\{w_{a(j)} \times \omega\left(w_{a(j)}, i\right): i=1, \ldots, n_{j}\right\}$ is the subset of $\Omega$ which is stacked over $w_{a(j)}$. Let $I_{q}(m)=\left\{t \in I:\left|t-m 2^{-q}\right| \leqq 2^{-q}\right\}$, then for an integer $q$ sufficiently large $I_{q}=\left\{I_{q}(m): m=0,1, \ldots, 2^{q}\right\}$ is a cover of $I$ which refines each of the covers $\omega_{j}, j=1, \ldots, k$, and also the cover $\gamma \beta(v)$. Let $p(0, i)=\left(x_{0}, i 2^{-q}\right)$ and $p(j, m)$ be a point in $X \times I$ such that $w_{a(j)} \times I_{q}(m) \subset \beta(v)_{p(j, m)}$ (note that $w_{a(j)} \times I_{q}(m) \subset w_{a(j)}$ $\times \omega\left(w_{a(j)}, i\right)$ for some integer $i$ and the latter set is a member of $\left.\Omega\right)$. Define $\beta(v)$-edge paths $\xi_{m}$ and $\eta_{m}: \xi_{m}=p(0, m), \ldots, p(k, m), \eta_{m}=p(0,0), p(0,1), \ldots, p(0, m)$ for $m=0, \ldots, 2^{q}$. Then $d_{m}=\eta_{m} * \xi_{m} * \eta_{m}^{-1}$ is a $\beta(v)$-edge loop in $X \times I$ based at $\left(x_{0}, 0\right)$; hence $H\left(d_{m}\right)$ is a $v$-edge loop in $Y$ based at $y_{1}$.

Consider the $v$-edge loop $\mu=H(p(0,0)), H(a(1), 0), H(p(1,0)), H(a(2), 0), \ldots$, $H(p(k-1,0)), H(a(k), 0), H(p(k, 0))$, where $a_{u}=a(1), \ldots, a(k)$ and $u=w \cap \alpha(v)$ were defined above. Since

$$
\begin{aligned}
H\left(\left(u_{a(j-1)} \cap u_{a(j)}\right) \times 0\right) & =f\left(u_{a(j-1)} \cap u_{a(j)}\right) \neq \varnothing, \\
H\left(\left(u_{a(j-1)} \cap u_{a(j)}\right) \times 0\right) & \subset H\left(\left(w_{a(j-1)} \cap w_{a(j)}\right) 0\right) \\
& \subset H\left(\beta(v)_{p(j-1,0)} \cap \beta(v)_{p(j, 0)}\right) \\
& \subset v_{H(p(j-1,0))} \cap v_{H(p(j, 0))}, \\
f\left(u_{a(j-1)}\right) \cap u_{a(j)} & \subset f\left(\alpha(v)_{a(j-1)} \cap \alpha(v)_{a(j)}\right) \\
& \subset f\left(\alpha(v)_{a(j)}\right) \subset v_{f(a(j))}=v_{H(a(j), 0)},
\end{aligned}
$$

we have $v_{H(p(j-1,0))} \cap v_{H(a(j), 0)} \cap v_{H(p(j, 0))} \neq \varnothing$ for $j=1, \ldots, k$; hence $\mu \sim H\left(d_{0}\right)$ $(\bmod v)$. Similarly $\mu \sim f\left(a_{u}\right)$, and by transitivity $H\left(d_{0}\right) \sim f\left(d_{u}\right)(\bmod v)$. It can also be shown that $H\left(d_{m}\right) \sim H\left(d_{m+1}\right)(\bmod v)$ for $m=0,1, \ldots, 2^{q}-1$ and that $H\left(\xi_{m}\right)$ $\sim g\left(a_{u}\right)(\bmod v)$. 
The integer $q$ was chosen so that the cover $\left\{I_{q}(m): m=0, \ldots, 2^{q}\right\}$ refines $\gamma \beta(v)$. Let $t(m) \in I$ be such that $I_{q}(m) \subset \gamma \beta(v)_{t(m)}$. Then the sequence $c=0, t(0), t(1), \ldots$, $t\left(2^{q}\right), 1$ is a $\gamma \beta(v)$-edge path in $I$ from 0 to 1 , and, by the proof of Theorem 3.5, $\boldsymbol{c} \sim \boldsymbol{b}_{\gamma \beta(v)}(\bmod \gamma \beta(v))$, hence $e(\boldsymbol{c}) \sim e\left(\boldsymbol{b}_{\gamma \beta(v)}\right)(\bmod \beta(v))$. The $\beta(v)$-edge path $\eta_{s}=p(0,0)$, $p(0,1), \ldots, p(0, s)$, where $s=2^{q}$, is partitioned in $e(c)$ and thus by Lemma 3.3, $\eta_{s} \sim e(\boldsymbol{c})$. By transitivity $\eta_{s} \sim e\left(\boldsymbol{b}_{\gamma \beta(v)}\right)$, whence $H\left(\eta_{s}\right) \sim H e\left(\boldsymbol{b}_{\gamma \beta(v)}\right)(\bmod v)$. Finally, from the preceding paragraph, $f\left(a_{u}\right) \sim f\left(a_{\alpha(v)}\right) \sim H\left(d_{s}\right) \sim H\left(\eta_{s}\right) * H\left(\xi_{s}\right) * H\left(\eta_{s}\right)^{-1}$ $\sim \mathrm{He}\left(\boldsymbol{b}_{\gamma \beta(v)}\right) * g\left(\boldsymbol{a}_{\alpha(v)}\right) * H e\left(\boldsymbol{b}_{\gamma \beta(v)}\right)^{-1}$. Hence the proof is complete.

Corollary 1. Let $(X, \tau)$ and $(Y, \sigma)$ be topological spaces such that $(X, P(\tau))$ and $(Y, P(\sigma))$ are coherently connected $((X, \tau)$ and $(Y, \sigma)$ path connected for example). If $(X, \tau)$ and $(Y, \sigma)$ are of the same homotopy type, then $E(X, P(\tau))$ is isomorphic to $E(Y, P(\sigma))$. (The base point may be suppressed for coherently connected spaces.)

COROLlary 2. If $(X, \tau)$ and $(Y, \sigma)$ satisfy the hypotheses of Corollary 1 with the functor $P$ replaced by the functor $Q$ (Example 2.3) and, in addition, are compact, then $E(X, Q(\tau)) \approx E(Y, Q(\sigma))$.

Corollary 3. If $(X, \tau)$ and $(Y, \sigma)$ satisfy the hypotheses of Corollary 1 with the functor $P$ replaced by the functor $U$ (Example 2.5) and, in addition, are compact, metric spaces, then $E(X, U(\tau)) \approx E(Y, U(\sigma))$.

The proofs of Corollaries 2 and 3 follow from Corollary 1 and Corollaries 1 and 2 of Theorem 6.3.

7. A Cech-type homology theory for pseudo-uniform spaces. Let $(X, C)$ be a pseudo-uniform space. For each $u \in C,\left\{u_{x}: x \in X\right\}$ is a cover of $X$ (by neighborhoods in the topology $T(C)$ ). An abstract simplicial complex $K(u)$ can be associated with each entourage $u \in C$ where the vertices $u_{x}$ of $K(u)$ are in $1: 1$ correspondence with points of $X$ and a finite subset $A=\left\{u_{x(1)}, \ldots, u_{x(n)}\right\}$ is a simplex in $K(u)$ if $\bigcap u_{x(i)}$ is nonvoid. Let $H_{j}(u)$ be the $j$ th homology group of $K(u)$ over an arbitrary coefficient group (Eilenberg and Steenrod [3, p. 166]). Let $u, v \in C$ with $u \subset v$. The identity function on $X$ induces a projection $p_{u v}: u \rightarrow v$ where $u$ and $v$ also denote the covers associated with these entourages. If $p_{u v}^{*}: H_{j}(u) \rightarrow H_{j}(v)$ is the induced homomorphism, then $\left\{\left(H_{j}(u), p_{u v}^{*}\right): u, v \in C\right\}$ is a projective system over $C$ directed by inclusion. Let $\hat{H}_{j}(X, C)$ be the projective limit. $\hat{H}_{j}(X, C)$ is the $j$ th Čech homology group of the pseudo-uniform space $(X, C)$.

Let $(X, \tau)$ be a topological space and let $C$ be a pseudo-uniformity for $X$ such that $T(C)=\tau$. The construction of $\hat{H}_{j}(X, C)$ differs from that of the usual Čech homology group $\hat{H}_{j}(X)$ in two respects: (1) the family of open covers generated by a base of open entourages for $C$ may be a proper subfamily of $\operatorname{Cov} X$. (2) The inclusion relation used to direct $C$ is contained in the refinement relation.

Proposition 7.1. Let $(X, C)$ be a pseudo-uniform space and

$$
\hat{H}_{j}\left(X, C^{(i)}\right)=\operatorname{proj} \lim \left\{\left(H_{j}, p_{u v}^{*}\right): u, v \in C^{(i)}\right\}
$$


over $C$ directed by inclusion. If $\hat{H}_{j}\left(X, C^{(r)}\right)=\operatorname{proj} \lim \left\{\left(H_{j}(u), p_{u v}^{*}\right): u, v \in C^{(r)}\right\}$ over $C$ directed by refinement, then $\hat{H}_{j}\left(X, C^{(r)}\right)$ is naturally isomorphic to $\hat{H}_{j}\left(X, C^{(i)}\right)$.

Proof. (The proof is similar to that of Theorem 6.3.)

THEOREM 7.2. If $(X, \tau)$ is a topological space and $P: \mathscr{T} \rightarrow \mathscr{S}$ is the functor of Example 2.2, then $\hat{H}_{j}(X)$ is naturally isomorphic to $\hat{H}_{j}\left(X, P(\tau)^{(i)}\right)$.

Proof. $P(\tau)^{(r)}$ is cofinal in $\operatorname{Cov} X$.

Corollary 1. If $(X, \tau)$ is a compact topological space and $Q: \mathscr{T} \rightarrow \mathscr{S}$ is the functor of Example 2.3, then $\hat{H}_{j}(X)$ is naturally isomorphic to $\hat{H}_{j}\left(X, Q(\tau)^{(i)}\right)$.

Proof. $(X, \tau)$ compact implies $Q(\tau)^{(r)}$ is cofinal in $P(\tau)^{(r)}$.

COROLlaRY 2. If $(X, \tau)$ is a compact, metric space and $U$ is the functor of Example 2.5 , then $\hat{H}_{j}(X)$ is naturally isomorphic to $\hat{H}_{j}\left(X, U(\tau)^{(i)}\right)$.

Proof. $(X, \tau)$ compact and metric implies that $U(\tau)^{(r)}$ is cofinal in $P(\tau)^{(r)}$.

The $u$-component $E_{u}\left(X, x_{0}\right)$ of the edge path group $E\left(X, C, x_{0}\right)$ is the edge path group of the simplicial complex $K(u)$ (Hilton and Wylie [4, p. 236]).

THEOREM 7.3. Let $\left(X, C, x_{0}\right)$ be a connected, pointed, pseudo-uniform space, and let $H_{1}(u)$ be the first homology group with integer coefficients of the complex $K(u)$ where $u \in C$. There exists an epimorphism $\phi_{u}: E_{u}\left(X, x_{0}\right) \rightarrow H_{1}(u)$ such that ker $\phi_{u}$ is the commutator subgroup of $E_{u}\left(X, x_{0}\right)$.

Proof. Let $C_{k}(u)$ be the free group generated by the ordered $k$-simplexes of $K(u)$. Then

$$
H_{1}(u)=\frac{\operatorname{ker} \partial_{0}}{\operatorname{Im} \partial_{1}} \text { where } C_{2}(u) \stackrel{\partial_{1}}{\longrightarrow} C_{1}(u) \stackrel{\partial_{0}}{\longrightarrow} C_{0}(u)
$$

If $a=a(1), \ldots, a(n)$ is a $u$-edge path in $X$, then $h(a)=\sum_{i=1}^{n-1}[a(i), a(i+1)]$ is a 1-chain in $C_{1}(u)$ where we write $[a(i), a(i+1)]$ for the 1 -simplex $\left[u_{a(i)}, u_{a(i+1)}\right]$. If $a$ is a $u$-edge loop at $x_{0}$ then $\partial_{0}(h(a))=\sum_{i=1}^{n-1}([a(i+1)]-[a(i)])=[a(n)]-[a(1)]=0$; hence $h(a) \in \operatorname{ker} \partial_{0}$. If $\boldsymbol{b}$ is also a $u$-edge path and is simply related to $a$, say for example, $\boldsymbol{b}=a(1), \ldots, a(j), x, a(j+1), \ldots, a(n)$ where $u_{a(j)} \cap u_{x} \cap u_{a(j+1)}$ is nonvoid, then $h(b)-h(a)=\partial_{1}([a(j), x, a(j+1)])$. From this we can conclude that if $\boldsymbol{b} \sim \boldsymbol{a}(\bmod u)$ then $h(b)$ is homologous to $h(a)$. Thus

$$
\phi_{u}: E_{u}\left(X, x_{0}\right) \rightarrow H_{1}(u)
$$

where $\phi_{u}([a])=[h(a)]$, is well defined; and furthermore, is clearly a homomorphism.

Let $z=\sum_{1}^{n} n(i)[r(i), s(i)]$ be a 1-chain in ker $\partial_{0}$. By allowing repetitions and replacing $-[a, b]$ by $[b, a]$, we may assume $n(i)=+1$ for $i=1, \ldots, n . \partial_{0}(z)=0$ implies $\sum[r(i)]=\sum[s(i)]$ and hence that $s(i)=r(j(i))$ for some integer $j(i) \in\{1, \ldots, n\}$ for $i=1, \ldots, n$. We conclude that $z$ has the form $z_{1}+\cdots+z_{k}$ where $z_{j}=$ $\sum_{1}^{m(j)}[x(i), y(i)]$ is such that $y(i)=x(i+1)$ for $i=1, \ldots, m(j)-1$ and $y(m(j))=x(1)$; 
that is to say, $a_{j}=x(1), \ldots, x(m(j))$ is a $u$-edge loop (not necessarily on $x_{0}$ ). Let $p(j)$ be the initial point of $a_{j}, j=1, \ldots, k$ and let $b_{j}$ be a $u$-edge path from $x_{0}$ to $p(j)$ ( $X$ is connected). Then $\boldsymbol{a}=\boldsymbol{b}_{1} * \boldsymbol{a}_{1} * \boldsymbol{b}_{1}^{-1} * \cdots * \boldsymbol{b}_{k} * \boldsymbol{a}_{k} * \boldsymbol{b}_{k}^{-1}$ is a $u$-edge loop on $x_{0}$ and $h(a)$ is homologous to $z$. Thus $\phi_{u}$ is an epimorphism.

Let $G$ be the commutator subgroup of $E_{u}$. Since $E_{u} / \operatorname{ker} \phi_{u}$ is commutative, $G \subseteq \operatorname{ker} \phi_{u}$. Let $[a] \in \operatorname{ker} \phi_{u}$ and let $z=\sum_{1}^{k} n(i)[r(i), s(i), t(i)]$ be a 2-chain in $C_{2}(u)$ such that $h(a)=\partial_{1}(z)$. Thus, if $[x, y]$ is a singular 1 -simplex appearing in the sum $\partial_{1} z-h(a)$, the sum of its coefficients is zero. Let $b_{1}$ be a $u$-edge path from $x_{0}$ to $r(i), i=1, \ldots, k$ and let $d_{i}=b_{i} *\{r(i), s(i), t(i)\} * b_{i}^{-1} . d_{i}$ is a null-equivalent loop on $x_{0}$, hence $e=a * d_{1}^{-n(1)} * d_{2}^{-n(2)} * \cdots * d_{k}^{-n(k)} \sim a$. If $e$ is the sequence of points $x(1), \ldots, x(p)$ then $f=\{x(1), x(2)\} *\{x(2), x(3)\} * \cdots *\{x(p-1), x(p)\} \sim e$. Let the multiplicity of a factor $\{x, y\}$ of $f$ be the number of times it occurs in $f$ minus the number of times $\{y, x\}$ occurs. Clearly, the multiplicity of a factor $\{x, y\}$ of $f$ is the sum of the coefficients of $[x, y]$ in the sum $\partial_{1} z-h(a)$, hence, is zero. Finally, let $g_{j}$ be a $u$-edge path from $x_{0}$ to $x(j), j=1, \ldots, p$ where $x(j)$ is a point of $\boldsymbol{e}$ and $\boldsymbol{g}_{j}=\boldsymbol{g}_{k}$ if $x(j)=x(k)$. Then $\boldsymbol{f} \sim \boldsymbol{g}_{1} *\{x(1), x(2)\} * \boldsymbol{g}_{2}^{-1} * \boldsymbol{g}_{2} *\{x(2), x(3)\}$ $* g_{3}^{-1} * \cdots * g_{p-1} *\{x(p-1), x(p)\} * g_{p}^{-1}=f_{1} * f_{2} * \cdots * f_{p-1}$, a product of $u$-edge loops each of whose multiplicity is zero. The class of the latter edge loop, hence that of $f, e$, and $a$ is in $G$. This completes the proof.

\section{BIBLIOGRAPHY}

1. N. Bourbaki, Topologie générale, Livre III, Chapitre 9, Hermann, Paris, 1963.

2. A. C. Carver, Topologies for function spaces, Doctoral Dissertation, New Mexico State Univ., University Park, N. M., 1963.

3. S. Eilenberg and N. Steenrod, Foundations of algebraic topology, Princeton Univ. Press, Princeton, N. J., 1952.

4. P. J. Hilton and S. Wylie, Homology theory. An introduction to algebraic topology, Cambridge Univ. Press, Cambridge, 1960.

5. J. L. Kelley, General topology, Van Nostrand, New York, 1955.

6. S. Lubkin, Theory of covering spaces, Trans. Amer. Math. Soc. 104 (1962), 205-238.

7. W. J. Pervin, Quasi-uniformization of topological spaces, Math. Ann. 147 (1962), 316-317.

8. H. Seifert and W. Threlfall, Lehrbuch der Topologie, Teubner, Leipzig, 1934.

9. A. Weil, Sur les espaces a structure uniforme et sur la topologie générale, Hermann, Paris, 1937.

New Mexico State University, University Park, New MeXico

IDAHO STATE UNIVERSITY, Pocatello, IDaho 